\title{
Justice et structure tragique : Webster
}

\section{Gisèle Venet}

Marie-Thérèse Jones-Davies (éd.)

\section{(2) OpenEdition Journals}

\section{Édition électronique}

URL : http://journals.openedition.org/shakespeare/124

DOI : 10.4000/shakespeare.124

ISSN : 2271-6424

Éditeur

Société Française Shakespeare

Édition imprimée

Date de publication : 1 novembre 1980

Pagination : 61-70

\section{Référence électronique}

Gisèle Venet, «Justice et structure tragique : Webster », Actes des congrès de la Société française Shakespeare [En ligne], 2 | 1980, mis en ligne le 01 novembre 2007, consulté le 27 avril 2019. URL http://journals.openedition.org/shakespeare/124; DOI : 10.4000/shakespeare.124 


\section{SOCIÉTÉ FRANC̣AISE SHAKESPEARE}

\section{ACTES DU CONGRĖS 1980}

DIRECTEUR DE LA PUBLICATION

M.T. Jones - Davies

JEAN TOUZOT Libraire - Editeur 38 , rue Saint-Sulpice 75278 PARIS CEDEX 061981 


\section{JUSTICE ET STRUCTURE TRAGIQUE : WEBSTER}

Un procès occupe l'acte central du Démon Blanc (The White Devil) pour condamner les amours illicites de Vittoria et du Duc Bracciano. Dans La Duchesse d'Amalfi (The Duchess of Malfi), c'est une juridiction implicite, dont rien ne dit qu'elle fût caduque, qui frappe d'illégalité le mariage des veuves; et la duchesse, très jeune veuve, se remarie en connaissance de cause.

On pourrait dès lors croire que Webster s'en remet à la justice pour organiser la. structure dramatique de ses deux tragédies. On pourrait croire que le soin revient «de droit» à la justice de tracer une frontière identifiable entre légitimité des initiatives privées et légalité des actions publiques, entre morale individuelle et éthique collective, la problématique frontière entre liberté et nécessité.

Mais Webster est l'un des derniers tragiques anglais. L'expérience est près de s'achever qui, par une esthétique en vertige, à la fois empirique et tragique, empirique parce que tragique, a conduit la sensibilité baroque hors de vieux continents vers de nouveaux ciels et de nouvelles terres. Rien en effet mieux que le tragique anglais n'aura «peint le passage», passage d'un monde clos à l'univers infini, passage d'une perception moniste du monde, pour laquelle tout était signe et réfléchissement, à une sensibilité dualiste qui ne dispose plus d'aucun repère pour explorer un devenir non balisé, «a wilderness where (we) shall find no path, nor friendly clue/to be (our) guide» (une étendue inexplorée où nulle trace, nulle balise familière, n'indiquera la route à suivre). Passage encore du monde de l'analogique, de la «proportion» dans la sphère du même à un univers ouvert, hypothétique, un univers pour lequel Pascal bientôt, au fragment 343 des Pensées, formulera l'évidence laconique : non pas «Dieu est mort», anachronisme absurde dans le contexte du tragique anglais comme dans le texte pascalien, mais «Le Grand Pan est mort», l'homme séparé ne trouve plus à lire l'ordre de la Surnature dans le Grand Livre de la Nature.

Pour Webster déjà, le miroir tendu à la nature ne reflète plus l'unité d'un cosmos, fût-il subtilement ou dangereuse- 
ment décentré comme dans shakespeare, mais l'égarement de la monade, corps-prison, réduite à n'habiter qu'une «motte de terre» exiguë, «a little turf of grass», comme pour Pascal l'homme se verra reléguer à «ce petit cachot, j'entends (précise-t-il) l'univers», univers infini réduit néanmoins à n'être que «the miserable compass of (our) prison» (limite dérisoire à notre prison). Univers de la séparation que láissaient présager les tempêtes shakespeariennes, tempêtes de l'esprit, «tempests in the mind». Et tout un mode de structuration tragique s'en trouve interdit - frappé de mutisme ou frappé de nullité comme on préfère.

Jusqu'ici des structures, à la fois mentales et esthétiques, avaient élu comme support privilégie du tragique le drame de vengeance dans lequel s'impliquaient et s'explicitaient des relations perçues comme contraignantes entre accident et nécessité, histoire et providence, ou encore justice immanente et justice transcendante. Quels que soient les délais, les retards, dans la durée contingente, le temps «image mobile de l'éternité» devenu mobilité indifférente à la durée, ramènerait le même au même. Une justice qui avait partie liée avec le temps, dans la durée contingente et en n'importe quel point accidentel de cette durée, nécessairement s'accomplirait. Dans La Tragédie Espagnole (The Spanish Tragedy), fondatrice du genre, l'un des personnages, victime innocente, Isabella,confie au temps le soin de faire justice de toutes les injustices : «Time is the author both of truth and right» (le temps crée de lui-même la vérité et la justice) «And time will bring this treachery to light» (et c'est au temps qu'incombe de dévoiler la forfaiture). Avec Hamlet, le mécanisme vengeur du temps menace de se gripper, «out of joint» et la prolifération démesurée de l'accidentel, du'contigent, bouffonnerie ou folie, est bien près d'étouffer la voix de la vengeance, bien près de priver la tragédie d'un dénouement, d'une structure de cohérence. Pourtant sous les durées éclatées, discordantes, un temps encore demeure latent, celui qui par «l'ironie du sort» ou «dramatic irony», ou encore "justice immanente», permet l'effet de retour, retour à la justice, retour à la légitimité qui cautionne cette justice. Dans les tragédies de Webster, on chercherait en vain des exemples de cette «ironie du sort» qui du moins aurait vectorisé le hasard, lui aurait prêté un sens en l'orientant vers un dénouement, aurait suggéré, si 
ténu soit-il, un lien structurel de cause à effet.

La solidarité entre justice et légitimité, dans la structure traditionnelle de la tragédie de vengeance, prédestinait des destins singuliers à œuvrer collectivement au rétablissement de la justice, quels qu'aient été les choix moraux, les options politiques, les stratégies contingentes des personnages concernés. Cette solidarité, dans l'œuvre de Webster, n'a plus d'actualité. Dans Le Démon Blanc comme dans La Duchesse d'Amalfi, si la justice est inique, ce n'est pas, ce n'est plus, parce que le pouvoir politique qui l'exerce est illégitime - ni le futur pape Monticelse, ni le duc de Florence dans le Démon Blanc, ni les frères de la Duchesse d'Amalfi, Ferdinand et le Cardinal, ne sont des usurpateurs; si la justice est inique c'est que le pouvoir est désormais sans référent, sans point fixe duquel dériver une ombre de nécessité, de légitimité transcendant l'ordinaire arbitraire de tout exercice du pouvoir. Antonio, nostalgique d'un monde caduc, au début de La Duchesse d'Amalfi rêve à un ordre stable, «the fix'd order» qui aurait régné à la Cour de France, pour mieux souligner semble-t-il le contraste avec les turbulences, les convulsions des Cours italiennes, ou la stagnation des valeurs inemployées, nauséabonde eau noire, «foul melancholy», dans laquelle s'agitent des malcontents. Mais l'allusion à un ordre ne fournit qu'un prologue au désordre. Nulle part ailleurs dans la pièce elle ne tissera «ce réseau de convergences, cette trame d'échos et de présences» qui selon Jean Rousset définissent une structure, et dans lesquels Shakespeare arrivait à saisir, par delà le pouvoir des princes du moment une légitimité infrangible. Nulle part ailleurs dans la tragédie elle ne suggère une grille de lecture dans laquelle surpendre, par-delà l'arbitraire d'une justice, la figure idéale de la justice.

Dans Le Démon Blanc et dans La Duchesse d'A malfi, justice et vengeance coexistent, sans implication mutuelle : l'une et l'autre soumises au seul arbitraire des choix et des stratégies individuelles, elles ne se trouvent que par hasard concourir à une même fin, fin dont on peut douter qu'elle vise un véritable dénouement. C'est le cas dans La Duchesse d'Amalfi où l'unité du récit pourrait reposer sur une stratégie sinueuse, efficace, omniprésente, celle de Bosola au service d'une vigilance obstinée et violente, celle de Ferdinand frère de la Duchesse, inquiet d'un remariage toujours 
possible de cette jeune veuve. Que l'interdit pesant sur ce remariage ne soit jamais formulé autrement que dans les convulsions passionnelles du Duc ou dans la froide duplicité du Cardinal peut à l'avance ôter toute sérénité à la justice si elle devait s'exercer - d'ailleurs l'arbitraire de la justice en a précédé tout exercice : Delio à propos de Ferdinand, nous met en garde : «the law to him/Is like a foul black cobweb to a spider» - il n'en reste pas moins que l'interdit est présent à la mémoire de la Duchesse comme interdit, et sa transgression volontaire, «dangerous venture», se fait en connaissance de cause. Mais Ferdinand, avant toute infraction, menaçait du poignard, non du juge, et sa vengeance, turbulente, échappant à tout contrôle, signe clinique de sa folie à venir, préviendra tout exercice de la justice. Webster n'en fait pas pour autant un «justicier légitime», il ne justifie sa vindicte par aucun motif : «Do not ask the reason: but be satisfied/ I say I would not», donne-t-il comme «raison» à Bosola de faire respecter l'interdit. Et après le meurtre de la Duchesse par Bosola, mission accomplie donc, le Duc (par duplicité ou chagrin qui l'égare, le texte maintient l'ambiguíté) se dénie toute légitimité à agir : «Was I her judge ?/ Did any ceremonial form of law/ Doom her to non-being ?» après avoir reproché à Bosola une stratégie trop parfaite, trop fidèle à ses ordres, après lui avoir fait grief de ne pas s'être interposé «entre son innocence à elle et sa vengeance à lui». Quant à cette glaciale efficacité de Bosola sur laquelle aurait pu se fonder la continuité d'un récit, elle n'interdit pas la discontinuité psychologique : le machiavel berné, l'homme du ressentiment, n'était qu'une éclipse - longue éclipse qui faillit couvrir la totalité de la pièce - dans une nature qu'on nous affirme «bonne». Cette option alternative, si elle permet à Bosola de s'improviser «justicier» pour tenter de défaire ce qu'au long de la trop longue éclipse il avait fait, ne rend pas davantage justice à la justice : «the office of justice is perverted quite/ When one thief hangs another». Elle ne rend pas non plus à la pièce son unité. L'unité, la seule unité possible,c'est le destin hors pair, hors frontière de la duchesse, cette exploration d'une «wilderness/ Where I shall find no path, nor friendly clue to be my guide», cette conquête d'une nouvelle innocence dans la culpabilité assumée, cette transgression hors des frontières de la morale collective; mais une 
unité qui ne se donne pas comme une structure d'intelligibilité, qui ne se donne pas comme une continuité, mais au contraire comme une discontinuité radicale, l'écart absolu avec le reste du récit, avec les stratégies et les intrigues qui finissent par la cerner, sans l'absorber jamais. La mort de la Duchesse, après un instant fulgurant où sur fond de charnier anonyme elle réaffirme une identité intangible - «I am Duchess of Malfi still» -, creuse encore l'écart : mort cruellement indifférente à l'interruption dans la durée -«Look thou givs't my little boy/ some syrup for his cold»mort pour laquelle il n'est pas de moment privilégié pour mourrir, pas plus qu'il n'y a de forme privilégiée de la mort - - I know death hath ten thousand several doors/ For men to take their exits》 - elle est ce moment hors du temps, ce moment de rupture après quoi les dénouements ultérieurs ne seront qu'une série de confuses méprises, des anti-dénouements. La mort de la Duchesse ne dénoue rien. Simplement après elle, la structure de la tragédie se défait, se complique, accélère la déperdition du sens. Pour la Duchesse, l'expérience de sa singularité, véritablement «existentielle», conduit à la frontière de la conscience de soi, conscience pure car conscience de rien, de rien de plus que la conscience qu'elle prend d'elle-même, solide et ténue comme le «cogito» à venir, pensée de rien autre que pensée qui se pense. Pure instantanéité dissociée de toute durée, de toute structure dans le temps, c'est la structure impossible.

Dans Le Démon Blanc, la justice se donne initialement comme exemplaire : Lodovico, en prologue, exemplairement condamné - «justly doomed»- ne conteste ni sa condamnation, l'exil, ni ses crimes. Il faut qu'il s'étonne de l'impunité que connaissent, pour les même délits, les puissants, dont Bracciano, pour que sa condamnation apparaisse plus conjoncturelle qu'exemplaire. Mais sa subite vocation de vengeur, à l'acte IV, ne semble aucunement naître d'un ressentiment contre «l'injustice» de cette condamnation, non plus que d'un sentiment de justice tiré de la condamnation de Vittoria, sa victime : il est absent du procès, absent de toutes les scènes litigieuses où Bracciano et Vittoria, «above law and above scandal», assurent leur bonheur contre toutes les règles. Bonheur continu et discontinu à la fois, bonheur qu'interrompt le procès intenté par Monticelso contre Vittoria, pour forcer à se compromettre le puissant et intou- 
chable Bracciano; mais bonheur qui reprendra plus tard, nouvelle excursion dans l'illégalité. Le procès lui-même, pourtant pièce centrale, n'a opéré qu'un arrêt momentané dans le récit des amours coupables. Sa justice rendue ne suggère aucun lien de nécessité structurelle qui le relierait au carnage - «massacre» - de la vengeance à l'acte $\mathrm{V}$, une vengeance dont Francisco se veut, si l'on peut dire, le légitime titulaire. Par ce procès, une double violation de la justice au contraire s'opère qui élimine le seul motif capable d'ordonner la tragédie en une forme déchiffrable, selon des normes identifiables, de «justifier» une vengeance : occasion d'un abus de pouvoir, il nous montre un Monticelso, juge et partie, conduisant à lui seul la procédure après élimination du représentant, d'ailleurs bouffon, de la justice, procédure dont il sait la fragilité légale, dont il avoue l'arbitraire : «Nous n'avons que des soupçons à retenir contre elle» ( We have naught but circumstances/ To charge her with»), procès corrompu - «corrupted trial» - que dénonce l'accusée elle-même, Vittoria : "You have ravish'd justice» (vous avez violé la justice). Mais façon plus subtile encore de la violer, ce procès offre à Webster et à Vittoria l'occasion d'oublier toute justice, d'annuler toute discrimination entre culpabilité et innocence : moment coupé de la continuité narrative, instant en soi, il oppose à l'arbitraire délictueux du pouvoir l'intégrité sans faille d'une volonté, coupable certes mais que cette intégrité, aux prises avec l'iniquité de la justice, rend paradoxalement innocente.

Ce que l'iniquité de la justice, de toute justice, révèle dans Le Démon Blanc c'est non l'absence de culpabilité, non le déni d'innocence, non la brimade d'un pouvoir arbitraire, mais la fin de toute norme, la reconnaissance de l'arbitraire comme seul mode d'être du pouvoir sans référence à un «droit divin», à une légitimité et une justice analogiquement identiques à la légitimité, à la justice divines. Symptomatiquement, à la même époque, Jacques Ier mène une bataille juridique obstinée pour recouvrer un «droit divin» qu'on ne lui accorde plus comme son droit «naturel». Et le juridique se révèle impuissant à restaurer l'alliance analogique, l'unité perdue. Jacques Ier ne sera qu'arbitrairement, si l'on peut dire, roi de «droit divin». C'est à Hobbes qu'il reviendra de prendre pleinement conscience du pouvoir fondateur de l'arbitraire, et de fonder sur l'arbitraire une nouvelle philo- 
sophie politique. En attendant, l'absence de fondement de la justice telle qu'arbitrairement elle s'exerce dans les tragédies de Webster aura eu pour effet de rendre aux volontés individuelles et aux modes collectifs de coercition leur indépendance. Liberté et nécessité ne se conjuguent plus sur le mode de l'unité, ne se structurent plus sur le mode de la contrainte. L'expérience tragique a changé de sens; l'individu au sens modernè du terme est peut-être en train de naître de cette expérience. D'une structure close, formelle, codifiée dans un temps lui-même clos, préformé, où les initiatives singulières se révélaient illusion de liberté, Webster est passé à une structure ouverte, scansion d'épisodes singuliers plus que caténation de moments solidaires, une structure dans laquelle l'informel domine, et que l'on a prise longtemps pour une défaillance du projet esthétique, une inaptitude à formuler avec cohérence une vision morale ou une visée métaphysique. En fait cette structure syncopée durablement reprochée à Webster, où l'instant l'emporte sur la continuité, ne fait que porter à un paroxysme ( au-delà duquel tout va se défaire et le tragique se résorber) ce qui était déjà le signe propre du théâtre jacobéen, la structure dissociative à l'origine du monde divisé de Chapman mais aussi d'une tragédie décentrée comme King Lear; King Lear, tragédie où deux récits coexistent en deux espaces disjoints, l'espace clos des intrigues enfermées dans l'enceinte des châteaux ou des camps militaires, et l'espace ouvert, l'espace liminaire au seuil d'une «wilderness», d'un univers proprement indescriptible, encore indéchiffré, celui d'errances multiples et parallèles, celui finalement de l'anonymat. Dans King Lear un combat pour la justice a bien lieu, combat singulier et «providentiel» s'il en fût, celui d'Edgar contre Edmund, mais qui ne «dénoue» qu'en apparence, et seulement la tragédie secondaire d'intrigues et d'usurpations, de justices codifiées qu'un jugement de Dieu, dans ce contexte familier, ne saurait désavouer. Mais cet apparent «dénouement» n'est là que pour détourner l'attention de tous, et la nôtre singulièrement, de l'absence de dénouement prévu pour l'autre tragédie, celle qui pour l'instant a relégué Lear et Cordelia hors scène dans ce «petit cachot», «miserable compass of a prison» où par hasard, par accident, on les oublie. En cette prison décentrée où pourtant l'essentiel arrive, ce qui arrive ne peut-être déchiffré, et n'arrive même ni trop tôt ni trop 
tard car après tout, on ne nous avait rien promis. A l'écart quelque chose arrive qui est la dilution du temps dans la contingence radicale, l'impossibilité désormais de confier au temps le nécessaire acomplissement d'une justice. La question épouvantée de Kent - «Is this the promis'd end ?» reste ouverte. Le hasard l'emporte sur la nécessité de fournir une réponse à notre attente, de tirer des conclusions, d'imposer une signification, d'édifier un système, de rendre la justice. Ne reste qu'un constat, constat déroutant, empirique et donc vertigineux, constat que la nature des choses ne fait plus référence à une idée de nature pour en garantir le sens; «la fin du monde» suggérée par la question ouverte de Kent pourrait bien être entre autres la fin d'une «idée de nature» qui jusqu'ici avait systématisé le réel.

Webster se situe dans la phase d'un nouveau tragique exploratoire d'où tout système, toute temporalité globale ou familière, d'où toute nécessité collective, semblent évacués. La mort elle-même y survient sans surprise, comme une donnée du réel, ici et maintenant indifféremment plutôt que tout à l'heure ou demain, tant il importe peu au dénouement le lieu et l'heure. Dans la succession de moments exceptionnels, suspendus hors du temps dans une durée indifférente, le moment de la mort n'est que le plus exceptionnel, car en lui se condensent « tout commencement et toute fin» comme Webster le fait dire à Flaminco mourant; en lui peut se revendiquer une conscience de soi qui pour n'être que d'un instant, n'en est pas moins indépassablement infrangible dans cet instant, instantanéité pure et complète, de diamant solitaire - le diamant, seule image vraiment obsédante chez Webster, le diamant qui seul peut entamer l'intégrité du diamant - «like diamonds, we are cut with our own dust». Artaud, ce maître du «théâtre de la cruauté», dira lui-même : «je me détruis jusqu'à ce que j'aie la preuve que c'est bien moi qui suis cela qui est moi, et non eux tous». Dans cet autre théâtre de la cruauté qu'est le tragique websterien, il semble bien que les personnages ne s'individualisent que dans l'acte singulier de la transgression ou de la mort, dans cet instant où ce qui les détruit les fait être, dans la fulguration dernière d'une conscience de la différence qui est conscience d'identité, d'intégrité d'identité, première forme moderne de la conscience de soi. Lodovico, personnage pourtant secondaire du Démon Blanc, compte 
pour rien la mort ou les tortures à venir car sa vengeance, même si la justice lui dénie toute validité, pour lui du moins institue sa différence : «I do glory yet/ That I can call this act mine own»: par la vengeance, il a pris conscience de ce qui est de lui, et non d'eux tous.

Théâtre de la Cruauté que cette audace implicite d'une déstructuration radicale, mentale et esthétique, à laquelle Webster nous contraint. Car c'est moins par le nombre des cadavres, et le raffinement de cruauté des mises à mort que cette tragédie, que ce tragique, accède au sommet de la cruauté baroque. Mais bien plutôt par la confrontation dans l'instant dernier de volontés adverses, dont rien ne peut entamer l'intégrité, la densité d'obscurs diamants à la transparence indéfinissable, cette cruauté nous situe audelà de toute compassion, au-delà de toute nostalgie, audelà de toute attente d'intelligibilité, de tout glissement vers un système,de toute sécurité héritée de structures familières, dans un temps et un espace encore inexplorées, ceux de notre modernité : intuition de l'instant et institution de la différence dans l'intuition instantanée d'une conscience de soi, par une radicalisation, une officialisation de la contigence comme seul horizon de ce tragique, «all coherence gone», toute providence absentée - tragique de la fin d'un monde, de la fin d'une illusion collective.

Gisèle VENET 